\title{
Compressed sensing with synchronized cardio- respiratory sparsity for free-breathing cine MRI: initial comparative study on patients with arrhythmias
}

\author{
Li Feng $^{1 *}$, Leon Axel ${ }^{1}$, Larry A Latson ${ }^{1}$, Jian X ${ }^{2}$, Daniel K Sodickson ${ }^{1}$, Ricardo Otazo ${ }^{1}$ \\ From 17th Annual SCMR Scientific Sessions \\ New Orleans, LA, USA. 16-19 January 2014
}

\section{Background}

Evaluation of myocardial function with MRI is challenging on patients with impaired breath-hold $(\mathrm{BH})$ capabilities or arrhythmias due to the difficulty of respiratory motion suspension and synchronization of cardiac cycles. Compressed sensing (CS) enables free breathing (FB) real-time cine imaging with improved spatiotemporal resolution, but conventional temporal sparsifying transforms do not account for respiratory motion, which limits its performance. In this work, we propose to acquire data continuously in FB using a golden-angle radial sampling scheme and reconstruct images with separated but synchronized cardiac and respiratory motion dimensions using self-detected motion signals. For patients with arrhythmias, both "normal" and "ectopic" cycles are reconstructed by sorting out cardiac cycles with different lengths. The performance of the proposed method was compared to Cartesian $\mathrm{BH}$ approach using retrospective ECG-gating in 9 patients.

\section{Methods}

Both $\mathrm{BH}$ and FB cine sequences (b-SSFP) were implemented on a 1.5T MRI scanner (Avanto, Siemens). Imaging parameters for $\mathrm{BH}$ cine were: spatial resolution $=1.8 \times$ $1.8 \mathrm{~mm}^{2}$, slice thickness $=8 \mathrm{~mm}$, TR/TE $=2.5 / 1.25 \mathrm{~ms}$, $\mathrm{FA}=55^{\circ}$. Imaging parameters for $\mathrm{FB}$ cine were: spatial resolution $=2 \times 2 \mathrm{~mm}^{2}$, slice thickness $=8 \mathrm{~mm}$, TR/ $\mathrm{TE} \approx 2.8 / 1.4 \mathrm{~ms}, \mathrm{FA}=70^{\circ}$. Both sequences achieved temporal resolution $\sim 30-40 \mathrm{~ms}$. Cardiac imaging was performed on 9 patients (mean age $=56$; 4 had normal sinus rhythm, 4 had arrhythmias including bigeminy PVCs, atrial fibrillation and Mobitz I, 1 was incapable of prolonged $\mathrm{BH}$ ). One short axis and one 4 chamber cine image set were acquired on each patient at $\sim 12-15$ s per slice. In FB cine imaging, central k-space positions (green dots, Figure 1a) were used to extract cardiac and respiratory signals from coils near the heart and diaphragm respectively (Figure 1b). Data were sorted and synchronized to separately reconstruct cardiac cycles of different lengths at different respiratory states. A multicoil CS approach was used to reconstruct the undersampled datasets, using a total variation constraint along both cardiac and respiratory dimensions. Images were randomized and blinded for radiologist evaluation (1-5; worst-best). Statistical analysis was performed to compare mean scores of $\mathrm{BH}$ and $\mathrm{FB}$ within two groups, one including normal sinus rhythm and the other including arrhythmias or impaired BH capability.

\section{Results}

Figure 1c shows $\mathrm{BH}$ and $\mathrm{FB}$ results on one patient with Mobitz I. The proposed approach produced better image quality due to the ability to differentiate "normal" and "ectopic" cycles (Table 1, group 2). For patients with normal sinus rhythm, both approaches produced good image quality (Table 1, group 1 ). Figure 1d shows FB cine reconstructions from "normal" and "ectopic" cycles. 


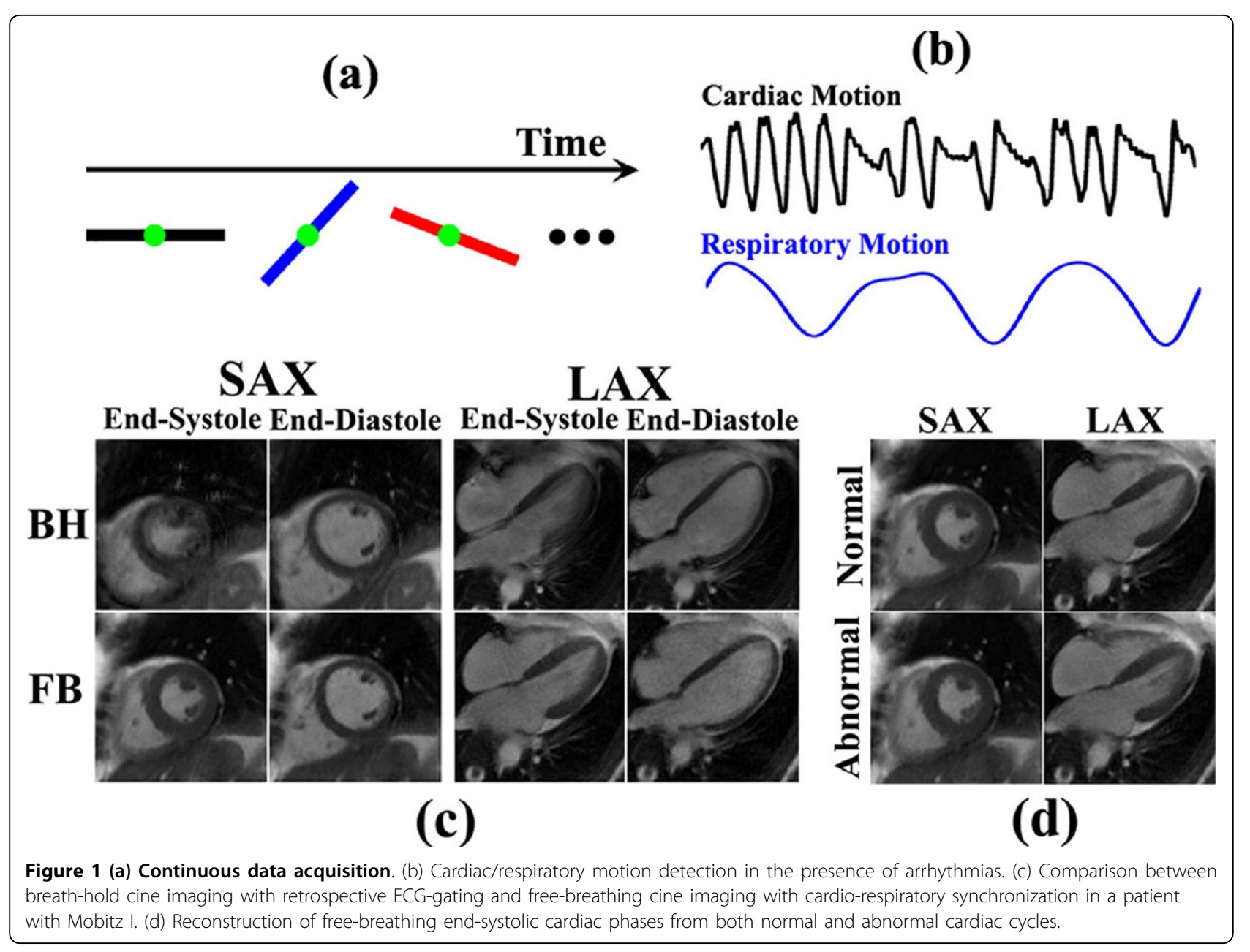

Table 1 Image quality comparison between breath-hold and free-breathing cine images.

\begin{tabular}{ccc}
\hline Technique & Group 1 & Group 2 \\
\hline BH & $3.75 \pm 0.46$ & $2.0 \pm 0.93$ \\
FB & $3.0 \pm 0.53$ & $3.1 \pm 0.74$ \\
\hline
\end{tabular}

Group 1 included patients with normal sinus rhythm and group 2 included patients with arrhythmias or impaired breath-hold capability.

\section{Conclusions}

Separation of cardiac and respiratory motion into different dimensions improves compressed sensing reconstruction for free-breathing imaging. Additional physiological information can be obtained by separately reconstructing cardiac cycles of different lengths.

\section{Funding}

National Institutes of Health: R01 EB000447.

\section{Authors' details}

${ }^{1}$ Bernard and Irene Schwartz Center for Biomedical Imaging, New York University School of Medicine, New York, New York, USA. ${ }^{2}$ Siemens Medical Solutions, New York, New York, USA.

Published: 16 January 2014

doi:10.1186/1532-429X-16-S1-017

Cite this article as: Feng et al:: Compressed sensing with synchronized cardio-respiratory sparsity for free-breathing cine MRI: initial

comparative study on patients with arrhythmias. Journal of Cardiovascular Magnetic Resonance 2014 16(Suppl 1):017. 